Leitbild für einen modernen Öffentlichen Gesundheitsdienst

\section{Konsens der länderoffenen Arbeitsgruppe zu einem Leitbild für einen modernen Öffentlichen Gesundheitsdienst}

Der Öffentliche Gesundheitsdienst ist in den letzten Jahren immer mehr in die gesundheitspolitische Diskussion zurückgekehrt. Anlass waren dabei zum einen Krisen, z. B. die EHEC-Krise oder die Flüchtlingskrise, in deren Bewältigung der ÖGD eine wichtige Rolle gespielt hat, zum anderen die zunehmenden Personalengpässe im ÖGD. In vielen Ländern können beispielsweise Arztstellen im ÖGD nur noch schwer besetzt werden. Hinzu kommt, dass der ÖGD auch bei der Umsetzung des Präventionsgesetzes unterstützend mitwirken soll. Parallel dazu fand im ÖGD selbst eine Diskussion über die inhaltliche Grundorientierung der Arbeit in den Gesundheitsämtern statt. Unstrittig ist, dass das Gesundheitsamt als reine Vollzugsbehörde den Anforderungen schon lange nicht mehr gerecht wird. Der ÖGD muss heute vielfältige Steuerungs- und Koordinationsaufgaben wahrnehmen, in Netzwerkstrukturen arbeiten und bei alldem, was der tut, auf hohem fachlichen, wissenschaftlich geprägten $\mathrm{Ni}$ veau agieren.

Die Gesundheitsministerkonferenz hat dem bereits 2016 durch einen weitreichenden Beschluss zur Stärkung des ÖGD Rechnung getragen. Ein Teilbeschluss hat die Entwicklung eines modernen Leitbilds für den ÖGD angeregt. Ein solches Leitbild wurde zwischenzeitlich durch eine länderübergreifende AG unter Beteiligung des BVÖGD erarbeitet. Die 91. GMK am 20./21.6.2018 hat nun den vorgelegten Leitbildentwurf begrüßt und empfiehlt „allen Akteuren des ÖGD, dieses Leitbild für die eigene Ausrichtung anzuwenden.“ (https://www.gmkonline.de/ Beschluesse. html?id=730\&jahr=).

Das Leitbild „Der Öffentliche Gesundheitsdienst - Public Health vor Ort“"ist darauf ausgerichtet, für die Arbeit in den Gesundheitsämtern eine konsistente, moderne Grundorientierung zu formulieren. Wichti- ge Aspekte dabei sind die Zusammenarbeit des ÖGD mit der Wissenschaft, die Orientierung auf Chancengleichheit und Gemeinwohnorientierung sowie eine ethisch verantwortliche Aufgabenerfüllung. Mit Blick auf die dazu notwendigen Qualifikationen wird die Bedeutung des Zusammenspiels von ärztlichen, sozial- und gesundheitswissenschaftlichen Qualifikationen im ÖGD betont. Im Folgenden dokumentieren wir dieses Leitbild als redaktionellen Beitrag.

Ihre Schriftleitung

\section{Leitbild für einen moder- nen Öffentlichen Gesund- heitsdienst - Zuständig- keiten. Ziele. Zukunft.}

\section{DER ÖFFENTLICHE GESUND- HEITSDIENST}

1. hat die öffentliche Verantwortung für die Gesundheit der Bevölkerung.

2. ist integraler Baustein des modernen Sozialstaats.

3. ist bürgernah und eingebunden in kommunale Strukturen.

4. orientiert sich an lokalen und globalen Herausforderungen.

5. ist gemeinwohlorientiert, ohne kommerzielle Interessen.

6. hat als Kernaufgaben Gesundheitsschutz, Gesundheitsförderung, Beratung und Information sowie Steuerung und Koordination.

7. nimmt hoheitliche Aufgaben wahr und arbeitet sozialkompensatorisch, planerisch und gestalterisch, um gesundheitliche Chancengleichheit und bestmögliche Gesundheit für alle zu ermöglichen (Public Health).

8. basiert auf medizinischen, insbesondere fachärztlichen, und sozial- sowie gesundheitswissenschaftlichen Qualifikationen.

9. arbeitet wissenschaftsbasiert und vernetzt.

10. ist ethisch reflektiert in Respekt vor der Würde des einzelnen Menschen.

\section{Der Öffentliche Gesundheits- dienst: Public Health vor Ort}

\section{Präambel}

Der Öffentliche Gesundheitsdienst (ÖGD) befindet sich in einem Wandel. Hoheitliche Schutz- und Überwachungsaufgaben werden um steuernde, partizipative und gesundheitsfördernde Tätigkeiten ergänzt. Dieses Leitbild will dabei insbesondere den Mitarbeiterinnen und Mitarbeitern in den Gesundheitsämtern Orientierung geben. Es sieht den ÖGD als einen zentralen Akteur der öffentlichen Sorge um die Gesundheit aller (Public Health) und schlägt eine Brücke zwischen Theorie und Praxis ebenso wie zwischen Gesundheitsschutz und Gesundheitsförderung.

\section{Der Öffentliche}

\section{Gesundheitsdienst im}

\section{modernen Sozialstaat}

Der Öffentliche Gesundheitsdienst(ÖGD) ist ein unverzichtbarer Teil eines modernen Sozialstaats. Er gehört neben der ambulanten und stationären Versorgung zur Basis des Gesundheitswesens. Der ÖGD nimmt im Rahmen der Daseinsvorsorge öffentliche Verantwortung für die Gesundheit der Bevölkerung wahr, ist zivilgesellschaftlich orientiert und arbeitet partnerschaftlich mit vielen Akteuren zusammen. Dies gilt für alle Ebenen des Öffentlichen Gesundheitsdienstes, von den Bundesbehörden über die Landesebene bis hin zu den Gesundheitsämtern.

\section{Leitorientierung im ÖGD}

Mit dem Wandel der Gesellschaft insgesamt geht auch ein Wandel des Selbstverständnisses und der Leitorientierung des Öffentlichen Gesundheitsdienstes einher. Neben seinen hoheitlichen Aufgaben muss er verstärkt modernen zivilgesellschaftlichen Erwartungen und sozialen Herausforderungen gerecht werden und in seiner $\mathrm{Ar}$ beitsweise zugleich auch dem wissenschaftlichen Anspruch an das Gesundheitswesen Rechnung tragen. Er strebt daher eine stärkere Berücksichtigung von Evidenz in Steuerungsprozessen an. Der ÖGD setzt sich für gesundheitliche Chancengleichheit ein. Dies prägt als Vision den ÖGD. Er arbeitet daher

- orientiert an prioritären Bedarfen der Bevölkerungsgesundheit, 
- ethisch reflektiert und in Respekt vor der Würde des einzelnen Menschen,

- mit Blick auf gesundheitliche Chancengleichheit,

- frei von kommerziellen Interessen,

- auf aktueller wissenschaftlicher Basis,

- bürgernah, vernetzt und partnerschaftlich mit vielen anderen Akteuren,

- multiprofessionell und interdisziplinär,

- ergebnisorientiert, verantwortlich und transparent.

Dabei bezieht sich der ÖGD konzeptionell auf Gesundheit als einen umfassenden körperlichen, psychischen und sozialen Zustand des Wohlbefindens. Er berücksichtigt dabei die wichtige Rolle einer gesundheitsförderlichen sozialen und ökologischen Lebenswelt.

\section{Kernaufgaben des ÖGD}

Der Öffentliche Gesundheitsdienst fördert und schützt die Gesundheit der Bevölkerung. Er trägt in einem arbeitsteiligen Gesundheitswesen dazu bei, Public Health in die Praxis umzusetzen. Die konkreten Aufgaben unterscheiden sich nach Maßgabe der jeweiligen Ländergesetze. Gemeinsame verbindende Schwerpunkte sind:

- Gesundheitsschutz (Hygiene, Infektionsschutz, einschließlich Impfen, umweltbezogener Gesundheitsschutz, Medizinalaufsicht, Ausbruchs- und Krisenmanagement),

- Beratung und Information, Begutachtung, Gesundheitsförderung und Prävention, niedrigschwellige Angebote und aufsuchende Gesundheitshilfen, insbesondere bei Personen mit besonderen Bedarfen (z. B. Kinder- und Jugendgesundheit, Mund- und Zahngesundheit, sozialmedizinische Aufgaben, wie Schwangerenberatung, Sozialpsychiatrie, Suchtberatung),

- Koordination, Kommunikation, Moderation, Anwaltschaft, Politikberatung, Qualitätssicherung (Gesundheitsberichterstattung, Gesundheitsplanung, Gesundheitskonferenzen, Öffentlichkeitsarbeit usw.).

Darüber hinaus werden in den einzelnen Ländern weitere Schwerpunktaufgaben durch den ÖGD wahrgenommen, z. B. gesundheitlicher Verbraucherschutz, subsidi- äre Versorgung besonderer Zielgruppen oder Umweltmedizin.

Dabei sind die traditionellen Aufgaben des Gesundheitsschutzes sowie der Fürsorge und die in den letzten Jahren verstärkt hinzu gekommenen planerischen und koordinativen Aufgaben gleichermaßen bedeutsam. Vom ÖGD werden heute sowohl hoheitliche Funktionen als auch das Gemeinwesen unterstützende und beratende Leistungen erwartet. Dies spiegelt sich auch auf europäischer Ebene in den dort definierten sog. Essential Public Health Operations (EPHOs) wider. Der ÖGD mit seinen multiprofessionellen Teams leistet einen wichtigen Beitrag, das Prinzip von „health in all policies “ in die Gremien der jeweiligen Handlungsebenen hineinzutragen. Der ÖGD unterstützt in bevölkerungsmedizinischen und gesundheitsplanerischen Fragen des Gesundheitswesens die jeweiligen politischen Entscheidungsträger, wodurch das Thema „Gesundheit“ im Bewusstsein der Akteure gestärkt wird und als Standortfaktor auf lokaler Ebene mehr Gewicht erhält.

\section{Arbeitsweisen, Ansätze und Funktionen}

Das genannte Aufgabenspektrum erfordert vom ÖGD verschiedene Kompetenzen. Seine Tätigkeit ist sowohl:

- risikoorientiert (pathogenetischer Ansatz) als auch ressourcenorientiert (salutogenetischer Ansatz),

- hoheitlich als auch partizipativ bzw. partnerschaftlich,

- bevölkerungs- bzw. sozialraum-als auch einzelfallbezogen.

Die Arbeitsweise umfasst dabei untersuchende, analysierende, planende, beratende, vernetzende, moderierende, koordinierende, steuernde, überwachende, kontrollierende und eingreifende Funktionen. Je nach Aufgabe sind dazu Kenntnisse aus unterschiedlichen medizinischen Fachgebieten, wie der Infektiologie, der Hygiene, der Kinder- und Jugendmedizin, der Toxikologie, der Arbeits-, Sozial- und Umweltmedizin, Zahnmedizin und Psychiatrie sowie auch Prävention und Gesundheitsförderung, der Epidemiologie, der Sozialen Ar- beit, der Psychologie und anderen Wissenschaftsbereichen essentiell.

Für die Erfüllung dieser Aufgaben arbeitet der ÖGD multiprofessionell, interdisziplinär und vernetzt. Medizinische - insbesondere fachärztliche - Qualifikationen bilden nach wie vor die Basis im Öffentlichen Gesundheitsdienst, sozialwissenschaftliche und gesundheitswissenschaftliche Qualifikationen sowie eine moderne Verwaltung sind heute jedoch ebenso unverzichtbar.

Wissenschaftlichkeit ist eine unverzichtbare Grundlage des ÖGD. Er benötigt eine enge Zusammenarbeit mit wissenschaftlichen Einrichtungen, insbesondere auch im Bereich Forschung und Lehre.

Zunehmende Bedeutung gewinnt darüber hinaus das systematische Zusammenspiel örtlicher Behörden des Öffentlichen Gesundheitsdienstes mit überregionalen Stellen (z. B. Landes- und Bundesbehörden), um Kompetenzen besser zu nutzen und Konzepte zu entwickeln und somit den ÖGD in seinen vielfältigen Aufgaben zu stärken.

\section{Stärken des ÖGD}

Der ÖGD hat traditionell einen bevölkerungsbezogenen Blickwinkel und arbeitet sozialkompensatorisch, gemeinwohlorientiert und frei von kommerziellen Interessen. Dies unterscheidet ihn von vielen anderen Akteuren des Gesundheitswesens. Wie kein anderer Akteur bündelt er zudem ein breites Spektrum an fachlicher Kompetenz aus unterschiedlichen wissenschaftlichen Disziplinen. Auf der örtlichen Ebene zeichnet er sich durch seine Bürgernähe und die Einbindung in die kommunalen Strukturen mit vielfachen Arbeitsbeziehungen zu anderen kommunalen Behörden (z. B. Schul-, Sozial-oder Jugendamt) und Institutionen aus. Er verfügt über Zugangsmöglichkeiten zu den verschiedenen Institutionen, Zielgruppen und Lebenswelten vor Ort (Krankenhäuser, Arztpraxen, Kindertagestätten, Schulen, Senioren- und Pflegeheime, Wohnbezirken, Stadt- oder Gemeindeteilen usw.). Neben den bevölkerungsmedizinischen Aufgaben wird der ÖGD bei Bedarf für besondere Personengruppen auch individualmedizinisch tätig und bietet aufsuchene Hilfen an. 


\section{Den ÖGD stärken}

Für die Weiterentwicklung des Öffentlichen Gesundheitsdienstes sind 3 Punkte von entscheidender Bedeutung:

- Der ÖGD braucht eine breite und nachhaltige politische Unterstützung aller Ebenen, von Kommune bis Bund. Es ist notwendig, die Personalentwicklung und Personalausstattung im ÖGD am Umfang seiner fachlichen Aufgaben auszurichten und nicht allein an finanzpolitischen oder verwaltungspolitischen Vorgaben.

- Die Rolle des Öffentlichen Gesundheitsdienstes im Zusammenspiel der Akteure im Gesundheitswesen muss entlang der genannten Kernaufgaben profiliert werden, insbesondere mit Blick auf die Stärkung der bevölkerungs- und sozialraumbezogenen Arbeit.

- Die Verbindung des Öffentlichen Gesundheitsdienstes mit der Wissenschaft muss sowohl in der Forschung als auch in der Aus- und Weiterbildung sowie in der Praxis gestärkt werden.

Ein starker ÖGD ist eine Voraussetzung für das Funktionieren des Public Health-Systems insgesamt. Dieser Feststellung der Wissenschaftsakademien ist in einem substanziellen Entwicklungsprozess Rechnung zu tragen. Es gilt, den Öffentlichen Gesundheitsdienst zukunftsfähig zu gestalten. Dazu will dieses Leitbild einen Beitrag leisten.

Dieses Leitbild wurde von der Gesundheitsministerkonferenz (GMK) 2016 angeregt und in einem offenen Diskussionsprozess mit dem ÖGD, Verbänden, zuständigen Gremien sowie der Wissenschaft konsentiert und von der GMK 2018 befürwortet.

Länderoffene Projektgruppe „Leitbild ÖGD“
Literatur

[1] Deutsche Akademie der Naturforscher Leopoldina e. V., acatech - Deutsche Akademie der Technikwissenschaften e. V., Union der deutschen Akademien der Wissenschaften e. V. Public Health in Deutschland. Strukturen, Entwicklungen und globale Herausforderungen. Halle, München, Mainz; 2015

[2] Grunow D, Grunow-Luther V. Der öffentliche Gesundheitsdienst im Modernisierungsprozess. Weinheim/ München: Juventa Verlag; 2000

[3] Kommunale Gemeinschaftsstelle für Verwaltungsmanagement (KGSt), Ziele, Leistungen und Steuerung des kommunalen Gesundheitsdienstes. KGSt-Bericht 11/1998. Köln

[4] Kuhn J, Wildner M, Zapf A. Der Öffentliche Gesundheitsdienst: Standortbestimmung mit hoffnungsvollem Ausblick. Deutsches Ärzteblatt 2012; 109: A 413-A 416

[5] Steen R. Soziale Arbeit im Öffentlichen Gesundheitsdienst. München: Ernst Reinhardt Verlag; 2005

[6] Teichert-Barthel U, Bornhofen B. Vom Physicus zum/zur Facharzt/-ärztin für Öffentliches Gesundheitswesen - Ein Berufsbild im Wandel. HygMed 2011; $36-1 / 2$

[7] Weltgesundheitsorganisation, Europäisches Regionalbüro. Europäischer Aktionsplan zur Stärkung der Kapazitäten und Angebote im Bereich der öffentlichen Gesundheit. Kopenhagen: WHO; 2012 